Proc. Indian Acad. Sci. (Earth Planet. Sci.), Vol. 95, No. 1, March 1986, pp. 83-89. (C) Printed in India.

\title{
The effect of filtering on drift and anisotropy parameters determined by full correlation analysis
}

\author{
B M VYAS, D V SARDESAI*, R K RAI, H CHANDRA $\uparrow$ and G D VYAS $\dagger$ \\ Department of Physics, Sukhadia University, Udaipur 313001, India \\ *Present address: Physics Department, vssD Post Graduate College, Kanpur 208002, India \\ $\uparrow$ Physical Research Laboratory, Navrangpura, Ahmedabad 380009, India \\ MS received 30 March 1985; revised 29 August 1985

\begin{abstract}
Ionospheric drift records obtained by the closely spaced receiver technique at Udaipur are subjected to low and high pass filtering with varying cutoff frequencies and the effects of filtering on various drift and anisotropy parameters as determined by full correlation analysis are studied. Limits in which filtering could be employed are suggested on the basis of results obtained.
\end{abstract}

Keywords. Ionospheric drift; full correlation analysis; spaced receiver method; filtering.

\section{Introduction}

Various methods have been developed for evaluating the drift parameters from spaced receiver records. The three methods used are (i) The similar fade method (Mitra 1949), which gives the apparent drift velocity $V_{a}$, and most commonly used earlier; (ii) The full correlation analysis (Briggs et al 1950; Phillips and Spencer 1955; Fooks 1965) mostly used nowadays that gives the apparent drift velocity, the true drift velocity $V$, characteristic velocity $V_{c}$ and various other parameters defining the characteristic ellipse representing the average size of the ground diffraction pattern namely the semiminor axis $b$, the axial ratio $r$ and the orientation of the major axis $\psi$; and (iii) cross spectral analysis, developed by Jones and Maude (1965) which gives the apparent drift velocity at different fading frequencies. The cross spectral method was developed to see if the diffraction pattern has wave motion. However, there are several possibilities which could result in dispersion in velocity like variation of velocity in the record or the presence of vertical gradients in velocity.

A different approach for the study of the variation of drift velocity with fading frequency was introduced by Sprenger and Schminder (1969). In this method the fading records are first filtered and then subjected to full correlation analysis. In principle one can thus study the apparent and the true drift velocity as well as other anisotropy parameters of the ground pattern as a function of the fading frequency by varying the cutoff frequency of the filter. Sprenger and Schminder (1969) reported an increase of the true drift velocity with the increase of the cutoff frequency of the high pass filter while the apparent drift velocity remained independent. Chandra and Briggs (1978) investigated theoretically the effect of low pass filtering assuming a Gaussian form of correlation functions and showed that the true drift velocity decreased with low pass filtering and therefore this need not be taken as evidence for dispersion. The filtering effect was seen only when $V_{c} / \pi f_{0} \xi_{1} \gg 1\left(\xi_{1}\right.$ is the scale size and $f_{0}$ the filter cutoff 
frequency) and for typical ionospheric conditions filter effects will be noted when cutoff frequency is less than about $1 \mathrm{~Hz}$. The apparent drift velocity, however, remained independent of filtering. In real cases of dispersion the correlation functions would be asymmetric and would show change in the apparent drift velocity with filtering. The theoretical investigations were compared and confirmed with experimental observations conducted at Buckland Park using the partial reflections. Thus it is obvious that filtering followed by correlation analysis cannot be used to determine true drift velocity at different fading frequencies.

Recently Chandra (1985) has studied theoretically the effect of low pass filtering on drift and anisotropy parameters and showed that while the true drift velocity $V$ decreases with filtering, the characteristic velocity $V_{c}$ decreases with filtering unless $V \gg V_{c}$ when $V_{c}$ shows an increase; resulting in an increase of the ratio $V_{c} / V$ with filtering since the decrease in $V$ is steeper than the decrease in $V_{c}$. The size (semi-minor axis) of the diffraction pattern was also shown to increase with filtering while the axial ratio would remain independent for near isotropic patterns but change for elongated patterns such as observed near the magnetic equator. The effects due to high pass filtering would be just the opposite as high pass filtering would result in narrowing down the correlation functions. The effect of filtering was shown, however, only when $V_{\mathrm{c}} / \pi f_{0} \xi_{1} \gg 1$ which worked out to be about $1 \mathrm{~Hz}$ for typical ionospheric conditions. A few samples of observational data recorded earlier at an equatorial station, Tiruchirapalli, confirmed these theoretical results.

In the present investigations the effect of low and high pass filtering is studied on a large number of spaced fading records obtained at a low latitude station Udaipur (Geog. lat. $24^{\circ} \mathrm{N}$ ). The primary aim of the study is to confirm the theoretical findings of Chandra (1985) and also to find out the limits in which filtering can be done without affecting the drift results.

\section{Observations and analysis}

About 80 records obtained at Udaipur during the year 1981- 82 have been analysed in this study. The experimental arrangement used has been described earlier (Rastogi et al 1978). The amplitude data has been digitized manually and fed to a computer for full correlation analysis. Record lengths between 1-2 minutes have been used for analysis with a sampling interval of 0.25 seconds. Filters of running mean type have been used with width varying between 0.50 to $10 \mathrm{sec}$. The cutoff frequency is determined as $f_{c}=1 / \tau_{f}$, where $\tau_{f}$ is the width of the filter. Hence in the present investigations cutoff

\section{Results}

The average variation of the various parameters computed from the full correlation analysis are plotted as a function of the cutoff frequency of the filter and shown in figures $1-5$. The results for both low pass and high pass fitters are shown in the same while in the case of high pass filters the amarked at the centre. It must be noted here that cutoff frequency of the filter, in the case of low of filtering increases with increase in the in the cutoff frequency of filters. 


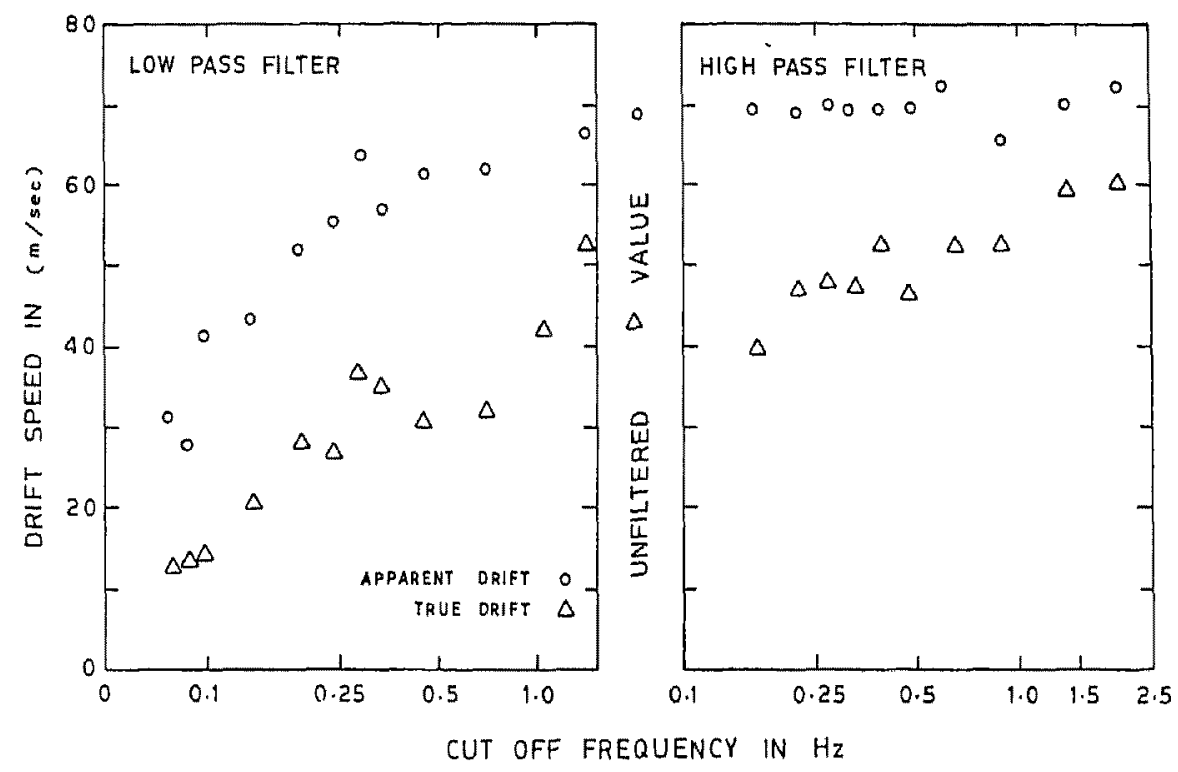

Figure 1. The variation of the apparent drift velocity $V_{a}$ and the true drift velocity $V$, determined by full correlation analysis, as a function of the cutoff frequency of the filter shown both for low pass and high pass filtering.



Figure 2. The variation of the characteristic velocity $V_{c}$ as a function of the cutoff frequency of the filter shown both for low pass and high pass filtering. 


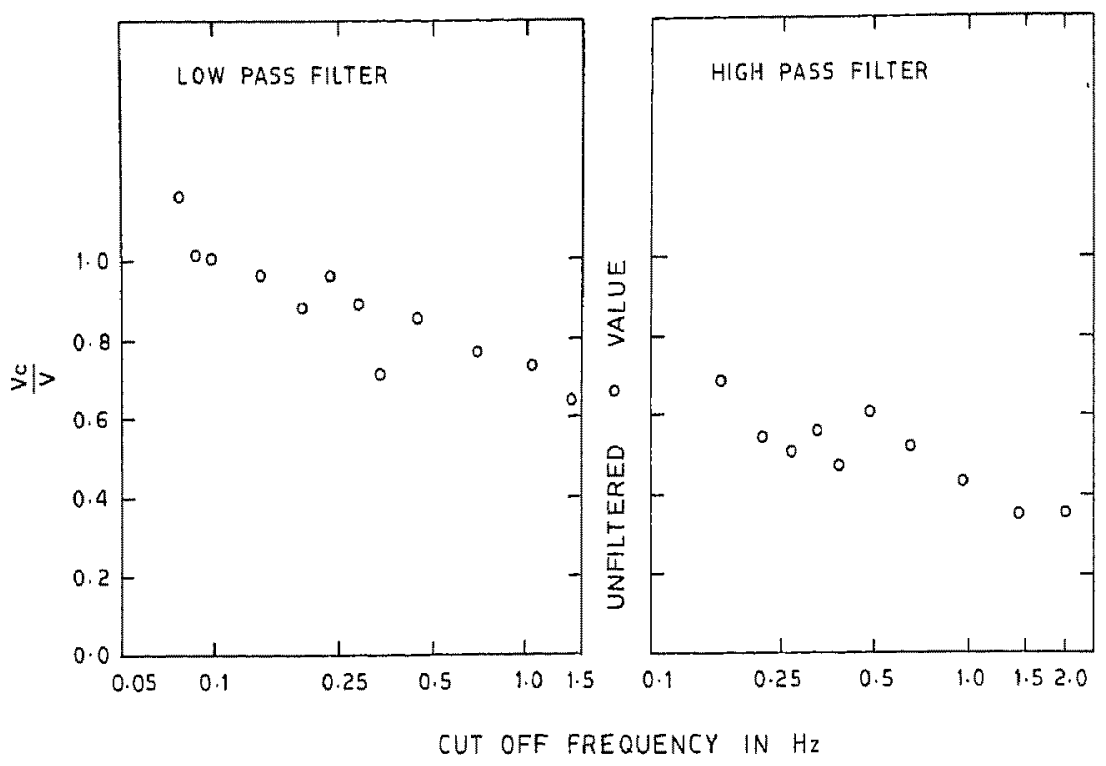

Figure 3. The variation of the ratio $V_{\mathrm{e}} / V$ as a function of the cutoff frequency of the filter shown both for low pass and high pass filtering.

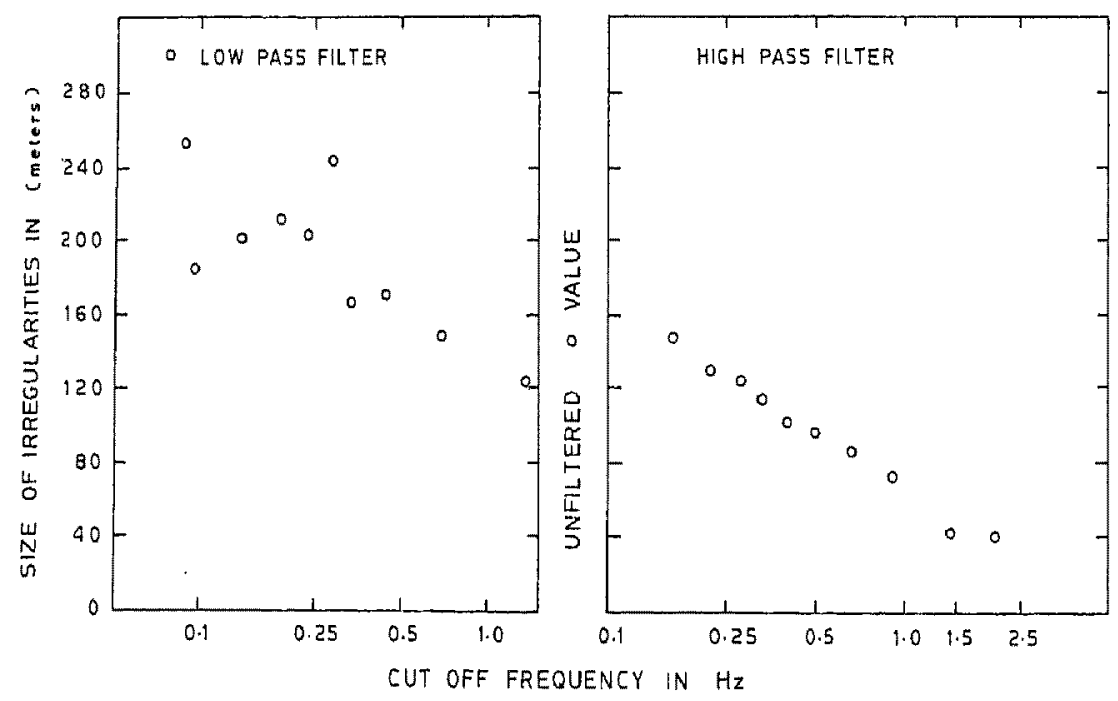

Figure 4. The variation of the scale size (b) of the irregularities determined by full correlation analysis as a function of the cutoff frequency of the filter shown both for low pass and high pass filtering. 


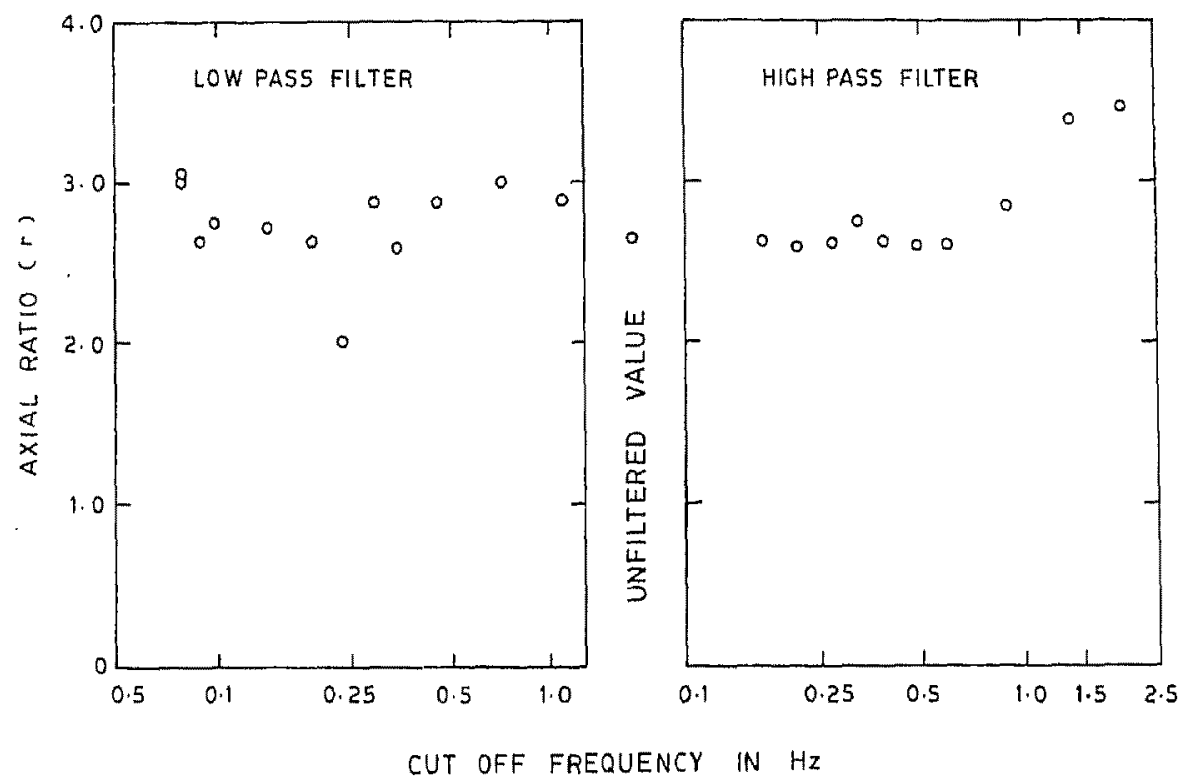

Figure 5. The variation of the axial ratio of the irregularities determined by correlation analysis as a function of the cutoff frequency shown both for low and high pass filtering.

The average variation of the apparent drift speed $V_{a}$ with cutoff frequency is shown in figure 1. It is seen that $V_{a}$ remains independent of the high pass filtering but decreases when low pass filtering is used. This decrease suggests the presence of dispersion. The variation of the true drift speed with filtering is also shown in the same figure. $Y$ t is found that the true drift speed $V$ increases with high pass filtering and decreases with low pass filtering. The unfiltered value of $43 \mathrm{~m} / \mathrm{s}$ decreases to about $13 \mathrm{~m} / \mathrm{s}$ with low pass filtering and approaches $V_{a}$ with high pass filtering. These results are similar to the results obtained earlier from experimental data (Sprenger and Schminder 1969; Chandra and Briggs 1978) and are also in agreement with the theoretical results of Chandra and Briggs (1978).

The average variation of the characteristic velocity $V_{c}$ with cutoff frequency is shown in figure 2. The value of $V_{c}$ does not show any significant variation with high pass filtering but decreases with low pass filtering, the decrease being from $29 \mathrm{~m} / \mathrm{s}$ to about $13 \mathrm{~m} / \mathrm{s}$. The average variation of the ratio $V_{c} / V$ shown in figure 3 indicates decrease of the ratio $V_{c} / V$ with high pass filtering and increase with low pass filtering. The unfiltered value of about 0.65 increases to more than 1.0 with low pass filtering and decreases to less than 0.4 with high pass filtering. Thus, while the effect of filtering (decrease) on parameters $V_{c}$ and $V$ is similar, the magnitude of effect is more pronounced for $V$ than for $V_{c}$. The unfiltered value of $V_{c} / V$ being about 0.6 , no increase in $V_{c}$ with filtering is expected on theoretical grounds.

The effect of filtering on the semi-minor axis $(b)$ of the characteristic ellipse is shown in figure 4. The scale size, $b$, decreases with high pass filtering and increases with low pass filtering. The average value of the scale size which is about $140 \mathrm{~m}$ increases to about $300 \mathrm{~m}$ with low pass filtering and decreases to a low value of about $40 \mathrm{~m}$ with high pass filtering. The average variation of the axial ratio $r$, of the characteristic ellipse is shown 
in figure 5. There is no significant variation in the value of axial ratio with filtering, the value lying between $2 \cdot 5$ to $3 \cdot 0$ on most cases.

\section{Discussion}

The effect of low pass filtering on the apparent and true drift velocity determined by full correlation analysis has been studied theoretically by Chandra and Briggs (1978). On theoretical grounds it is expected that the true drift velocity would decrease with low pass filtering while apparent drift velocity remains independent. Chandra (1985) has studied theoretically the effect of low pass filtering on characteristic velocity $V_{c}$, the ratio $V_{c} / V$, scale size $b$ as well as axial ratio $r$. According to Chandra (1985) there would be a decrease in the characteristic velocity $V_{c}$ with low pass filtering unless $V \gg V_{c}$, when there is an increase in $V_{c}$, and the ratio $V_{c} / V$ would always increase. The scale size would increase with low pass filtering. The limiting value of the increase given by $\left[\left(V^{2}+V_{c}^{2}\right) / V_{c}^{2}\right]^{1 / 2}$. The results in the present investigations are in conformity with these theoretical investigations. Theoretical investigations by Chandra (1985) have also shown that for isotropic irregularities the axial ratio would remain independent of the filtering. Since the axial ratio at Udaipur is about $2 \cdot 5$, one would not expect any significant effect of the filtering. This result is also observed in the experiment results presented here. For a low latitude station Udaipur, the limits in which filtering could be employed comes out to be about $\frac{1}{2} \mathrm{~Hz}$ (cutoff frequency).

Cross spectral analysis has been applied to a large number of $F$-region records at Udaipur in an earlier study (Sardesai et al 1983) and it was noted that about $21 \%$ cases showed velocity increasing with fading frequency (positive dispersion). No dispersion in velocity was noted in about $18 \%$ cases and nearly $46 \%$ cases showed velocity varying randomly with fading frequency. Similar patterns were also found for both $E$ and $F$ region observations at an equatorial station Tiruchirapalli (Vyas et al 1980). Positive dispersion has been thought to arise due to the variation of velocity within the record or due to the presence of vertical gradients in velocity (Felgate and Golley 1971; Huag and Petterson 1970; Chandra 1979). A comparison of the dispersion results by cross spectral and filtering method showed similar variation of velocity with the fading frequency (Vyas and Chandra 1985). The eighty records studied here were also analysed by the cross spectrum method. Positive dispersion was noted on about $22 \%$ of records while $8 \%$ of the records showed negative dispersion. Approximately $25 \%$ of the records did not show any dispersion and the rest of the records showed no consistent variation of the velocity with fading frequency. The variation of the drift and anisotropy parameters as a function of the filter cutoff frequency was then studied separately for the four categories of dispersion as seen by the cross spectral method. Except for the apparent drift velocity the result for other parameters were similar to those shown in figures 1-5 which are averages of the data. The apparent drift velocity, for the positive dispersion group, increased with the cutoff frequency of the high pass filters and decreased with the decrease of the cutoff frequency of low pass filters (increasing filtering). For the group showing negative dispersion the results were just the opposite i.e. the apparent drift velocity showing an increase with low pass filtering and a decrease with cutoff frequency of high pass filters. For the groups showing 'no dispersion' and 'random dispersion' the effect of filtering on the apparent drift velocity were similar. Hence the filter method can only study the variation of the apparent drift velocity with fading 
frequency. The variations in other parameters arise by the nature of the analysis itself and have no physical significance. The cross spectral method has limitations in that it can determine only the apparent drift velocity and due to this reason it appeared earlier that the filter method could be used to study various drift and anisotropy parameters at different fading frequencies. However, both the theoretical work of Chandra (1985) as well as the present experimental investigations suggest that the results obtained by the filter method may lead to wrong interpretation if the limitations of the method are not taken into account. In actual experimental data some sort of filtering is always present, imposed by the finite length of data and the sampling interval. In addition, detrending is necessary sometime. However, when using filters for detrending, care must be taken that the filter cutoff frequency chosen satisfies the conditions that parameters to be studied do not change appreciably. This is given by the condition $V_{\mathrm{c}} / \pi f_{0} \xi_{1} \gg 1$ and for the ionospheric fading data for a low latitude station like Udaipur is about $\frac{1}{2} \mathrm{~Hz}$.

\section{Acknowledgements}

One of us (BMv) is thankful to the UGC, New Delhi for a fellowship.

\section{References}

Briggs B H, Phillips G J and Shinn D H 1950 Proc. Phys. Soc. (London) B63 106

Chandra H 1979 Proc. Indian Acad. Sci. A88 41

Chandra H 1985 Indian J. Radio Space Phys. (Communicated)

Chandra H and Briggs B H 1978 J. Atmos. Terr. Phys. 40541

Felgate D G and Golley M G 1971 Aust. J. Phys. 24397

Fooks G F 1965 J. Atmos. Terr. Phys. 27979

Huag A and Petterson H 1970 J. Atmos. Terr. Phys. 32397

Jones D and Maude A D 1965 Nature (London) 206177

Mitra S N 1949 Proc. Inst. Electr. Eng. 96441

Phillips G J and Spencer M 1955 Proc. Phys. Soc. 868481

Rastogi R G, Chandra H, Singhal K F, Rai R K, Janve A V and Kumar V 1978 Indian J. Radio Space Phys. 76

Sardesai D V, Rai R K, Vyas G D and Chandra H 1983 Indian J. Radio Space Phys. 1218

Sprenger K and Schminder R 1969 J. Atmos. Terr. Phys. 311085

Vyas G D, Chandra H and Rastogi R G 1980 Indian J. Radio Space Phys, 930

Vyas G D and Chandra H 1985 Indian J. Radio Space Phys. (Communicated) 